\title{
Patient-specific detection of perfusion abnormalities combining within-subject and between-subject variances in Arterial Spin Labeling
}

\author{
Camille Maumet $^{\mathrm{a}, \mathrm{b}, \mathrm{c}, \mathrm{d}, *}$, Pierre Maurel ${ }^{\mathrm{a}, \mathrm{b}, \mathrm{c}, \mathrm{d}}$, Jean-Christophe Ferré ${ }^{\mathrm{a}, \mathrm{b}, \mathrm{c}, \mathrm{d}, \mathrm{e}}$, Béatrice Carsin ${ }^{\mathrm{e}}$, Christian Barillot $^{\mathrm{a}, \mathrm{b}, \mathrm{c}, \mathrm{d}}$ \\ ${ }^{a}$ University of Rennes 1, Faculty of medecine, F-35043 Rennes, France \\ ${ }^{b}$ INSERM, U746, F-35042 Rennes, France \\ ${ }^{c}$ CNRS, IRISA, UMR 6074, F-35042 Rennes, France \\ ${ }^{d}$ Inria, VISAGES project-team, F-35042 Rennes, France \\ ${ }^{e} \mathrm{CHU}$ Rennes, Department of Neuroradiology, F-35033 Rennes, France
}

\begin{abstract}
In this paper, patient-specific perfusion abnormalities in Arterial Spin Labeling (ASL) were identified by comparing a single patient to a group of healthy controls using a mixed-effect hierarchical General Linear Model (GLM). Two approaches are currently in use to solve hierarchical GLMs: (1) the homoscedastic approach assumes homogeneous variances across subjects and (2) the heteroscedastic approach is theoretically more efficient in the presence of heterogeneous variances but algorithmically more demanding. In practice, in functional magnetic resonance imaging studies, the superiority of the heteroscedastic approach is still under debate. Due to the low signal-to-noise ratio of ASL sequences, within-subject variances have a significant impact on the estimated perfusion maps and the heteroscedastic model might be better suited in this context.

In this paper we studied how the homoscedastic and heteroscedastic approaches behave in terms of specificity and sensitivity in the detection of patient-specific ASL perfusion abnormalities. Validation was undertaken on a dataset of 25 patients diagnosed with brain tumors and 36 healthy volunteers. We showed evidence of heterogeneous within-subject variances in ASL and pointed out an increased false positive rate of the homoscedastic model. In the detection of patient-specific brain perfusion abnormalities with ASL, modeling heterogeneous variances increases the sensitivity at the same specificity level.
\end{abstract}

Keywords: Arterial Spin Labeling, Hypo-perfusion, Hyper-perfusion, General Linear Model, Within-subject variance, Heteroscedasticity

\section{Introduction}

Arterial Spin Labeling (ASL), a Magnetic Resonance Imaging (MRI) technique introduced in the early 1990's, permits the measurement of the level of perfusion through a quantitative index: the cerebral blood flow (CBF). Contrary to standard perfusion imaging, including Positron Emission Tomography (PET) and Single Photon Emission Computed Tomography (SPECT) in nuclear medicine, or Dynamic Susceptibility weighted Contrast (DSC) in MRI, ASL is completely non-invasive and does not require the injection of an exogenous contrast agent. In the context of this paper, we aim at outlining areas of abnormal perfusion in the perfusion map of a subject of interest by comparison to a group of healthy controls.

The most widespread approach to compare voxel-wise maps in neuroimaging is the massively univariate General Linear Model (GLM). To detect differential patterns between two groups with repeated measurements a sub-

\footnotetext{
${ }^{*}$ Corresponding author

Email address: contact@camillemaumet.com (Camille Maumet)
}

type of GLM is employed: a mixed-effect hierarchical twosample t-test with two levels: subject and group. In this context, two variance components are of interest: the withinsubject variance (or the measurement error, estimated from the repeated ASL acquisitions of a single subject) and the between-subject variance.

In the functional MRI community, where similar statistical models are applied, two approaches are currently in use to solve hierarchical GLMs. On the one hand, the homoscedastic approach, also termed "summary statistics" (Penny and Holmes, 2004), or referred as "ordinary least square estimation" (Mumford et al., 2006, Mumford and Nichols, 2009) or "conventional group analysis" (Chen et al. 2012), assumes homogeneous within-subject variances or negligible within-subject variances by comparison to between-subject variance. On the other hand, the heteroscedastic approach, also referred as "full mixedeffect" (Friston et al., 2005; Thirion et al., 2007; Poldrack et al., 2011), "mixed-effect model" (Chen et al., 2012) or "weighted least square estimation" (Mumford et al. 2006: Mumford and Nichols, 2009) models heterogeneous within-subject variances. There is indeed a large panel 
of homonyms to refer to these two approaches, in current practice they are also sometimes referred as "mixed-effect" and "random-effect" approaches (Lindquist et al., 2012). However, in a statistical sense both the homoscedastic and the heteroscedastic approaches are mixed-effect models. That is why, in the context of this paper, we chose to term the two approaches "homoscedastic model" and "heteroscedastic model" to identify them according to their main difference: the homoscedasticity (constant within-subject variance across subjects) assumption.

The homoscedastic model is theoretically less efficient in the presence of heterogeneous within-subject variances. However, in practice, the true variance components are unknown and the superiority of the heteroscedastic model is therefore questioned. Whether modeling heterogeneous variances should be preferred over the homoscedastic model is still under discussion in the fMRI community. Several authors outlined the benefits of heteroscedastic models including (Worsley et al., 2002, Beckmann et al., 2003, Woolrich et al., 2004, Mumford et al., 2006 Mériaux et al., 2006, Thirion et al., 2007, Chen et al., 2012). However, Mumford and Nichols (2009) showed that the homoscedastic approach is still valid with near optimal sensitivity in the context of one-sample t-tests. Nonetheless, the same authors acknowledged that, in two-sample t-tests, appropriate modeling of heterogeneous within-subject variances might be crucial (Mumford and Nichols, 2009, Poldrack et al., 2011). Recently the conclusions regarding onesample t-tests were revisited leading to opposite conclusions (Chen et al., 2012). In the different software packages currently available to deal with fMRI data both approaches are represented: SPM ${ }^{1}$ favors the homoscedastic approach (Holmes and Friston, 1998) while FSL ${ }^{2}$ (Woolrich et al. 2004), AFNl ${ }^{3}$ (Cox, 1996) and fmristat ${ }^{4}$ (Worsley et al., 2002) use the heteroscedastic model.

In this paper, we focus on quantitative detections of pathological brain perfusion abnormalities at the individual level. To this aim, we employ and compare two GLMbased models: the homoscedastic and heteroscedastic approaches. We test whether the assumptions underlying the homoscedastic approach are verified in pulsed ASL datasets. We furthermore study how the homoscedastic and heteroscedastic approaches behave in terms of specificity and sensitivity in the detection of patient-specific perfusion abnormalities.

A quantitative validation is performed on a dataset of 25 patients diagnosed with brain tumors. The model of normal perfusion is computed out of the data of 36 healthy volunteers.

Section 2 begins with a presentation of the homoscedastic and heteroscedastic models employed in the detection of patient-specific brain perfusion abnormalities with

\footnotetext{
1 http://www.fil.ion.ucl.ac.uk/spm

2 http://fsl.fmrib.ox.ac.uk/fsl/fslwiki

3 http://afni.nimh.nih.gov/afni

4 http://www.math.mcgill.ca/keith/fmristat
}

ASL. Section 3 presents the datasets under study: the acquisition and pre-processing are described. Then, in section 4, the experiments designed to test the assumptions of the homoscedastic model and measure sensitivity and specificity are described. The results are presented in section 5. Section 6 gives a discussion and concludes.

\section{Theory}

In this section, the homoscedastic and heteroscedastic models used in the context of patient-specific detection of perfusion abnormalities in ASL are presented. In the standard approach, the GLM is defined for each voxel, which is why this approach is often termed "massively univariate". Without loss of generality to the multi-voxel procedure, we will consider the case of a single voxel in the remainder of this section. The voxel index is therefore omitted. In the context of this paper, we focus on a subtype of GLM: a one-versus-many mixed-effect two-sample t-test. As several measurements are available for each subject, a hierarchical model is defined with 2 levels: subject and group.

\subsection{Subject level (First level)}

In ASL, at the subject level, the data under study is a $4 \mathrm{D}$ volume of CBF maps containing $r$ volumes (1 per repeated acquisition). The observations are therefore repeated measurements of the same underlying value. The subject level is therefore defined by:

$$
\boldsymbol{Y}_{\boldsymbol{s}}=\left[\begin{array}{c}
1 \\
\vdots \\
1
\end{array}\right] \beta_{s}+\boldsymbol{\epsilon}_{\boldsymbol{s}}
$$

where $\boldsymbol{Y}_{\boldsymbol{s}}$ is a vector of length $r$ containing the observations at the given voxel and $\beta_{s}$ is the subject parameter to be estimated. Assuming Gaussian noise of the errors, each element of $\boldsymbol{\epsilon}_{\boldsymbol{s}}$ follows a normal distribution: $\epsilon_{s}^{v} \sim$ $\mathcal{N}\left(0, \sigma_{s}^{2}\right)$. While in fMRI temporal autocorrelation must be accounted for (Aguirre et al. 1997), in ASL, thanks to the subtraction process between control and labeled scans, we can reasonably assume white noise (Aguirre et al. 2002, Mumford et al., 2006). The subject parameter $\beta_{s}$ is thus estimated by Ordinary Least Squares (OLS):

$$
\hat{\beta}_{s}=\frac{1}{r} \sum_{i=1}^{r} y_{s, i}
$$

where $y_{s, i}$ is the $\mathrm{i}^{\text {th }}$ element of vector $\boldsymbol{Y}_{\boldsymbol{s}}$. Similarly, the sampling variance of $\hat{\beta}_{s}$ is estimated by:

$$
\widehat{\operatorname{Var}}\left(\hat{\beta}_{s}\right)=\frac{\hat{\sigma}_{s}^{2}}{r} \quad \text { where } \hat{\sigma}_{s}^{2}=\frac{1}{r-1} \sum_{i=1}^{r}\left(y_{s, i}-\hat{\beta}_{s}\right)^{2}
$$




\subsection{Group level (Second level)}

Given a group of $n$ subjects, without loss of generality we assume that subjects 1 to $n-1$ are part of the control group and subject $n$ is the patient of interest. Then, the one-versus-many second-level model is defined by:

$$
\left[\begin{array}{c}
\hat{\beta}_{1} \\
\vdots \\
\hat{\beta}_{n-1} \\
\hat{\beta}_{n}
\end{array}\right]=\left[\begin{array}{cc}
1 & 0 \\
\vdots & \vdots \\
1 & 0 \\
0 & 1
\end{array}\right]\left[\begin{array}{c}
\beta_{\text {controls }} \\
\beta_{\text {patient }}
\end{array}\right]+\gamma_{G_{C}} .
$$

The error term $\gamma_{G_{C}}$ contains two combined sources of variations: the error measurement on the subject parameters (also termed within-subject variance) and the between-subject variance. Each element of $\gamma_{G_{C}}$ therefore follows a normal distribution: $\gamma_{G_{C}}^{s} \sim \mathcal{N}\left(0, \sigma_{G}^{2}+\frac{\sigma_{s}^{2}}{r}\right)$.

Linear combinations of the group parameters can be calculated using a particular contrast. Here we are interested in the patient versus control group contrast:

$$
b=\left[\begin{array}{ll}
1 & -1
\end{array}\right]\left[\begin{array}{l}
\beta_{\text {controls }} \\
\beta_{\text {patient }}
\end{array}\right]=\beta_{\text {controls }}-\beta_{\text {patient }} .
$$

Two approaches have been proposed in the neuroimaging literature to solve the system $\sqrt{3}$ and find an estimate of the patient versus control group contrast, $\hat{b}$, and its associated sampling variance, $\widehat{\operatorname{Var}}(\hat{b})$.

The homoscedastic model is based on the assumption that the within-subject variance is either negligible by comparison to the between-subject variance (i.e. $\sigma_{s}^{2} \ll$ $\sigma_{G}^{2}, \forall 1 \leq s \leq n$ ) or, roughly constant across subjects (i.e. $\left.\sigma_{s}^{2} \approx \sigma_{S U B}^{2}, \forall 1 \leq s \leq n\right)$. Then each element of $\gamma_{G_{C}}$ follows a normal distribution:

$$
\gamma_{G_{C}}^{s} \sim \mathcal{N}\left(0, \sigma_{G_{C}}^{2}\right)
$$

where $\sigma_{G_{C}}^{2}$ is the combined within- and between-subject variance. Depending on the assumption, we have $\sigma_{G_{C}}^{2} \approx$ $\sigma_{G}^{2}+\frac{\sigma_{S U B}^{2}}{r}$ or $\sigma_{G_{C}}^{2} \approx \sigma_{G}^{2}$ and the combined within- and between-subject variance is therefore constant across subjects. The sphericity assumption (no heteroscedasticity and no autocorrelation) hence holds and the system (3) is then solved by OLS. In the heteroscedastic model, heterogeneous variances are accounted for. Due to the nonsphericity of the measurement errors (as a consequence of heteroscedasticity), the system (3) is solved by using a weighted least square.

For a detailed calculation of the homoscedastic and heteroscedastic estimates, the interested reader is referred to previous developments of this methodology in the fMRI literature (for example Mumford and Nichols (2009); Beckmann et al. (2003)). Tables 1 and 2 provides a summary of the final estimates for the homoscedastic and heteroscedastic models. Table 1 gives the estimates of the control group $\left(\hat{\beta}_{\text {controls }}\right)$ and patient $\left(\hat{\beta}_{\text {patient }}\right)$ effects along with their sampling variances $\left(\widehat{\operatorname{Var}}\left(\hat{\beta}_{\text {controls }}\right)\right.$ and $\left.\widehat{\operatorname{Var}}\left(\hat{\beta}_{\text {patient }}\right)\right)$. Table 2 provides the estimate of the control group versus patient effect $(\hat{b})$ along with its sampling variance $(\widehat{\operatorname{Var}}(\hat{b}))$, both estimates are obtained by combination of the control group and patient estimates:

$$
\begin{gathered}
\hat{b}=\hat{\beta}_{\text {controls }}-\hat{\beta}_{\text {patient }} \\
\widehat{\operatorname{Var}}(\hat{b})=\widehat{\operatorname{Var}}\left(\hat{\beta}_{\text {controls }}\right)+\widehat{\operatorname{Var}}\left(\hat{\beta}_{\text {patient }}\right)
\end{gathered}
$$

If the data are homoscedastic then it can easily be proven that the heteroscedastic model reduces to the homoscedastic model. However, in the presence of heteroscedasticity, the heteroscedastic model has two main advantages by comparison to the homoscedastic model:

1. In the control group, observations with high withinsubject variances are downweighted in order to provide a more efficient estimate of the control group parameter, $\hat{\beta}_{\text {controls }}$.

2. While with the homoscedastic model the sampling variance of the patient group estimate, $\widehat{\operatorname{Var}}\left(\hat{\beta}_{\text {patient }}\right)$, depends solely on variance estimations performed in the control group, the heteroscedastic sampling variance takes advantage of both the control group (estimation of $\sigma_{G}^{2}$ ) and the patient (estimation of $\sigma_{n}^{2}$ ) data.

The impact on the efficiency of the control group estimate (point 1) might be subtle and leads to no substantial improvement in one-sample t-tests if the homoscedasticity assumption is not overly altered (Mumford and Nichols. 2009). However, the impact on the variance estimate of the patient parameter (point 2) can be large if the withinsubject variance of the patient of interest is very different from the control subjects. In ASL studies, the large influence of within-subject variance has been described in ( $\mathrm{Vi}-$ viani et al. , 2009). Furthermore, patients are known to be less cooperative than control subjects which could potentially lead to within-subject variance inflation due to increased movement.

\subsection{Hypothesis testing}

Under the null hypothesis:

$$
H_{0}: \beta_{\text {controls }}=\beta_{\text {patient }},
$$

the estimated patient versus control group contrast, $\hat{b}$, divided by its estimated sampling standard deviation $(\widehat{\operatorname{Var}}(\hat{b}))^{\frac{1}{2}}$ follows a t-distribution with $n-1$ degrees of freedom.

A probability under the null hypothesis can therefore be calculated for each voxel with:

$$
P(X<x) \text { where } X=\frac{\hat{b}}{\sqrt{\widehat{\operatorname{Var}}(\hat{b})}} \sim \mathcal{T}_{n-1},
$$

and $x$ is the value taken by $X$ at the voxel of interest. Equation (8) gives the probability to have a hyperperfusion, similarly, hypo-perfusions can be detected by substituting $P(X<x)$ by $P(X>x)$. The thresholding of this probability map gives the detections. 
Table 1: Control group and patient estimates along with their sampling variances for the homoscedastic and heteroscedastic models.

\begin{tabular}{ccccc} 
& $\hat{\beta}_{\text {controls }}$ & $\hat{\beta}_{\text {patient }}$ & $\widehat{\operatorname{Var}}\left(\hat{\beta}_{\text {controls }}\right)$ & $\widehat{\operatorname{Var}}\left(\hat{\beta}_{\text {patient }}\right)$ \\
\hline Homoscedastic & $\frac{1}{n-1} \sum_{s=1}^{n-1} \hat{\beta}_{s}$ & $\hat{\beta}_{n}$ & $\frac{\hat{\sigma}_{G_{C}}^{2}}{n-1}$ & $\hat{\sigma}_{G_{C}}^{2}$ \\
\hline \multirow{2}{*}{ Heteroscedastic } & $\frac{1}{\sum_{j=1}^{n-1} \frac{1}{\hat{\sigma}_{G}^{2}+\hat{\sigma}_{j}^{2}}} \sum_{s=1}^{n-1} \frac{1}{\hat{\sigma}_{G}^{2}+\hat{\sigma}_{s}^{2}} \hat{\beta}_{s}$ & $\hat{\beta}_{n}$ & $\frac{1}{\sum_{s=1}^{n-1} \frac{1}{\hat{\sigma}_{G}^{2}+\hat{\sigma}_{s}^{2}}}$ & $\hat{\sigma}_{G}^{2}+\hat{\sigma}_{n}^{2}$ \\
\hline
\end{tabular}

Table 2: Control group versus patient estimate along with its sampling variance for the homoscedastic and heteroscedastic models.

\begin{tabular}{ccc}
\hline & $\hat{b}$ & $\widehat{\operatorname{Var}}(\hat{b})$ \\
\hline Homoscedastic & $\frac{1}{n-1} \sum_{s=1}^{n-1} \hat{\beta}_{s}-\hat{\beta}_{n}$ & $\hat{\sigma}_{G_{C}}^{2}\left(\frac{1}{n-1}+1\right)$ \\
\hline Heteroscedastic & $\frac{1}{\sum_{j=1}^{n-1} \frac{1}{\hat{\sigma}_{G}^{2}+\hat{\sigma}_{j}^{2}}} \sum_{s=1}^{n-1} \frac{1}{\hat{\sigma}_{G}^{2}+\hat{\sigma}_{s}^{2}} \hat{\beta}_{s}-\hat{\beta}_{n}$ & $\frac{1}{\sum_{s=1}^{n-1} \frac{1}{\hat{\sigma}_{G}^{2}+\hat{\sigma}_{s}^{2}}}+\hat{\sigma}_{G}^{2}+\hat{\sigma}_{n}^{2}$ \\
\hline
\end{tabular}

\section{Materials}

This section starts with a presentation of the datasets under study in 3.1. The pre-processing steps applied to the data before the statistical analysis are then described in 3.2 .

\subsection{Data acquisition}

25 patients diagnosed with brain tumors and 36 healthy volunteers were involved in this study. One control subject and four patients were excluded because of strong borderzone signs (Zaharchuk et al., 2009). The final dataset therefore included 21 patients $(13$ males, 8 females, age: $55.2 \pm 14.1$ years) and 35 healthy volunteers (16 males, 19 females, age: $27.7 \pm 6.4$ years).

Data acquisition was performed on a $3 \mathrm{~T}$ Siemens Verio MR scanner with a 32-channel head-coil. Patients were scanned in the context of clinical practice. The imaging protocol included a 3D T1-weighted anatomical sequence (T1w) (TR: $1900 \mathrm{~ms}$, TE: $2.27 \mathrm{~ms}$, FOV: $256 \mathrm{~mm}$ $\times 256 \mathrm{~mm} \times 176 \mathrm{~mm}$, flip angle: $9^{\circ}$, resolution: $1 \mathrm{~mm}$ $\times 1 \mathrm{~mm} \times 1 \mathrm{~mm}$ ), a PICORE Q2TIPS sequence (Wong et al., 1998) with crusher gradients (TR: $3000 \mathrm{~ms}$, TE: $18 \mathrm{~ms}$, FOV: $192 \mathrm{~mm} \times 192 \mathrm{~mm}$, flip angle: $90^{\circ}$, resolution: $3 \mathrm{~mm} \times 3 \mathrm{~mm}$, slice thickness: $7 \mathrm{~mm}$, inter-slice gap: $0.7 \mathrm{~mm}$, TI: $1700 \mathrm{~ms}, \mathrm{TI}_{w d}$ : $700 \mathrm{~ms}, 60$ repetitions, mSENSE parallel imaging with accelerating factor of 2). In addition to these sequences, the patients also underwent a 3D T1w post gadolinium (T1w-Gd) sequence (TR: $1900 \mathrm{~ms}$, TE: $2.27 \mathrm{~ms}$, flip angle: $9^{\circ}$, FOV: $250 \mathrm{~mm} \times 250 \mathrm{~mm} \times 176 \mathrm{~mm}$, resolution: $1 \mathrm{~mm} \times 1 \mathrm{~mm}$ $\times 1 \mathrm{~mm}$ ) and a 2D T2w FLAIR sequence (TR: $9000 \mathrm{~ms}$, TE: $90 \mathrm{~ms}$, FOV: $220 \mathrm{~mm} \times 199.4 \mathrm{~mm}$, flip angle: $150^{\circ}$, resolution: $0.69 \mathrm{~mm} \times 0.69 \mathrm{~mm}$, slice thickness: $4 \mathrm{~mm}$ ).
Out of the 22 patients, 17 subjects also underwent a DSC sequence (GRE EPI, TR: $1500 \mathrm{~ms}$, TE: $30 \mathrm{~ms}$, FOV: $230 \mathrm{~mm} \times 230 \mathrm{~mm}$, flip angle: $90^{\circ}$, in plane resolution: $1.8 \mathrm{~mm} \times 1.8 \mathrm{~mm}$, slice thickness: $4 \mathrm{~mm}$, inter-slice gap: $1.2 \mathrm{~mm})$.

\subsection{Pre-processing}

\subsubsection{CBF estimation with $A S L$}

Image pre-processing was performed using SPM8 (Statistical Parametric Mapping 8, Wellcome Department of Imaging Neuroscience, University College, London) in Matlab R2012a (Mathworks, Natick, MA). The anatomical image of each subject was segmented, bias corrected and normalized into the ICBM-452 T1 template space using the unified segmentation (Ashburner and Friston, 2005). The normalization was performed using an affine linear transformation followed by a non-linear transformation based on discrete cosine transform functions (default SPM normalization scheme). A subject-specific anatomical brain mask was created, excluding voxels with less than $50 \%$ of brain tissue in subsequent statistical analyses. A sixparameter rigid-body registration of the ASL volumes was carried out in order to reduce undesired effects due to subject motion. Rigid coregistration onto the whole-brain anatomical map was then performed based on mutual information. The average of unlabeled volumes was used to estimate the geometrical transformation to apply to each volume. Pair-wise subtraction of the control and labeled scans was then computed. A standard kinetic model (Buxton et al. 1998) was applied in order to get ASL CBF, according to the following equation:

$$
f=6000 \times \frac{\lambda \Delta M}{2 M_{0} \alpha T I_{w d} \exp ^{-\left(T I+i d x_{s l} * T I_{s l}\right) / T 1_{b}}}
$$


where $f$ is the $4 \mathrm{D}$ CBF map in $\mathrm{mL} \cdot 100 \mathrm{~g}^{-1} \cdot \mathrm{min}^{-1}, M_{0}$ the acquired $\mathrm{M}_{0}$ map (first volume of the ASL series), $\lambda$ the blood/tissue water partition coefficient, $\alpha$ the labeling efficiency, $\Delta M$ the $4 \mathrm{D}$ perfusion-weighted map, $T I=1.7 \mathrm{~s}$ the inversion time (Ferré et al. 2012), $i d x_{s l}$ the slice index ( 0 for the first slice), $T I_{s l}=0.045 \mathrm{~s}$ the readout time for one slice, $T I_{w d}=0.7 \mathrm{~s}$ the temporal width of the bolus, $T I_{b}$ the $\mathrm{T} 1$ of blood. According to Wang et al. (2011), we assumed: $\lambda=0.9 \mathrm{~mL} \cdot \mathrm{g}^{-1}, T I_{b}=1.5 \mathrm{~s}$ and $\alpha=0.95$. The 6000 factor allows the conversion from $\mathrm{mL} \cdot \mathrm{g}^{-1} . \mathrm{s}^{-1}$ to $\mathrm{mL} .100 \mathrm{~g}^{-1} \cdot \mathrm{min}^{-1}$ which is the standard unit for CBF. We bring the attention of the reader to the fact that, contrary to what is usually done in ASL pre-processing, $\Delta M$ represents the set of perfusion-weighted maps (one volume per repetition) instead of a single perfusion-weighted map obtained by averaging across the repetitions. This is necessary in order to allow for the measurement of the within-subject variance.

Spatial normalization parameters estimated during the segmentation step were then applied to the T1 and ASL CBF maps in order to normalize the subjects into a common template space. Normalisation in intensity was then applied to each ASL CBF map in order to reduce the intersubject variability Aslan and Lu (2010). The normalisation parameter was calculated as the average CBF in grey matter similarly to Petr et al. (2012).

\subsection{2. $C B F$ estimation with $D S C$}

The DSC images were processed using MR manufacturer software by manually choosing an arterial input function to calculate: the CBF, cerebral blood volume, and mean transit time maps. The method is based on a deconvolution algorithm as described in (Ostergaard et al. 1996). Similarly to ASL, DSC CBF maps were coregistered on anatomical maps and spatially normalized.

\subsection{Ground truth}

Based on clinical knowledge, we used a semi-automatic procedure that took advantage of the complementary anatomical (T1w-Gd, T2w FLAIR) and perfusion (DSC) information to get an estimation of the ground truth.

True positives. In order to get an estimation of the ground truth, we implemented a method inspired by the hotspot technique Noguchi et al. 2008) on the DSC CBF map. To this aim, the tumor was first segmented using a semiautomated method based on the T2w and T1w-Gd images and visually inspected by an expert neuro-radiologist. Then, we compared the tissue segmented as part of the tumor to its controlateral counterpart in the DSC CBF map. Voxels overtaking the lower and upper deciles were identified as potential hypo- and hyper-perfusions. Each potential perfusion abnormality was then visually inspected by an expert neuro-radiologist and manually corrected if needed. Special care was taken in order to avoid inclusion of hyper-perfusions related to the presence of arteries.
Due to its low SNR, ASL is not well suited to measure low levels of perfusion (Wintermark et al. , 2005). That is why, we focused on hyper-perfusions for sensitivity estimation. Hypo-perfusions were nevertheless retained for specificity calculations.

Out of the 17 patients included in this study who underwent a DSC sequence, 9 presented hyper-perfusions, 16 hypo-perfusions and 8 both.

False positives. According to clinical knowledge, in the absence of metastasis, the perfusion abnormalities should be confined to the affected tissue (tumor and oedema) identifiable on T1w-Gd and T2w. The proportion of nonaffected tissue detected as a perfusion abnormality was used as a measure of the false positive rate.

In the control group, in which no detections were expected, an additional estimate of the specificity was calculated by leave-one-out cross-validation.

\section{Methods}

In 4.1 the implementation of the heteroscedastic model employed in this paper is described. Then, in 4.2, the experiments undertaken in order to test the assumptions of the homoscedastic model and to compare the homoscedastic and heteroscedastic models are presented.

\subsection{Implementation choice for the heteroscedastic model}

In this paper, we computed the estimate of the betweensubject variance, $\hat{\sigma}_{G}^{2}$, using a recent computationally efficient approach available in the MEMA function of the AFNI software package. This algorithm is based on the method of moments and the restricted maximum likelihood. The estimation method is then selected depending on the data observed at the voxel level (Chen et al., 2012). It is however worth noting that alternative publicly available algorithms, such as FLAME1 in FSL, are also suited to compute this kind of heteroscedastic approach.

\subsection{Evaluation framework}

\subsubsection{Validity of the assumptions of the homoscedastic model}

The homoscedastic model makes the assumption that within-subject variance is either negligible by comparison to between-subject variance, or roughly constant across subjects. In order to test these assumptions, we performed two experiments.

Negligible within-subject variance. First, following Chen et al. (2012), we measured the proportion of total variability that occurred within subjects with the following index, defined at each voxel $v$ for each subject $s$ :

$$
\lambda_{s, v}=\frac{\hat{\sigma}_{s, v}^{2}}{\hat{\sigma}_{G, v}^{2}+\hat{\sigma}_{s, v}^{2}}
$$


(a)

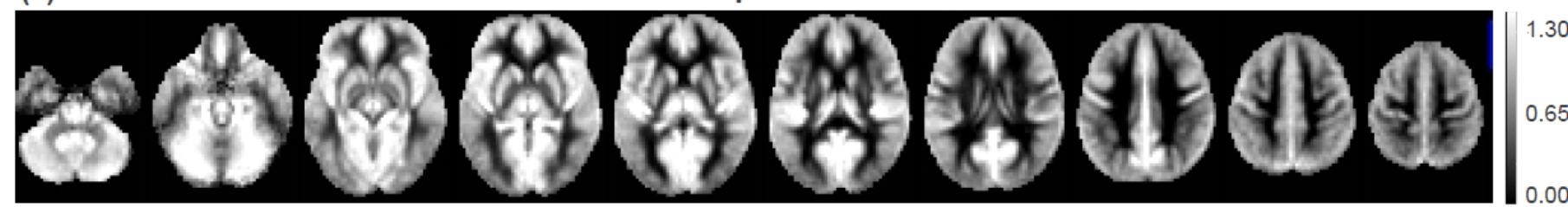

(b) Combined within- and between-subject standard deviation (homoscedastic model)

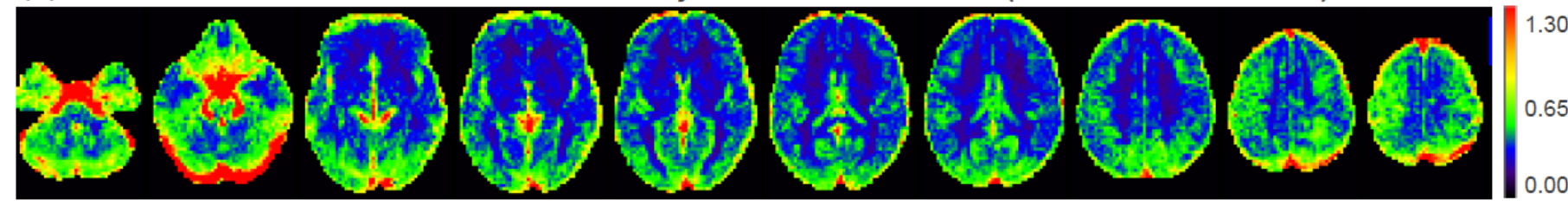

(c) Between-subject standard deviation (heteroscedastic model)

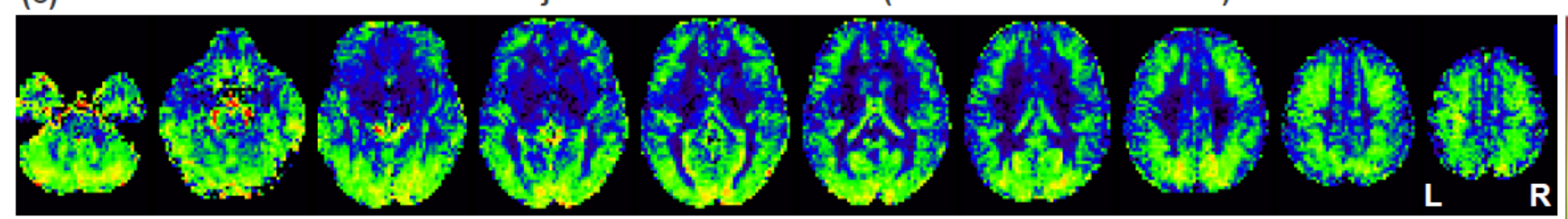

(d) Root-square of the average within-subject variance (heteroscedastic model)

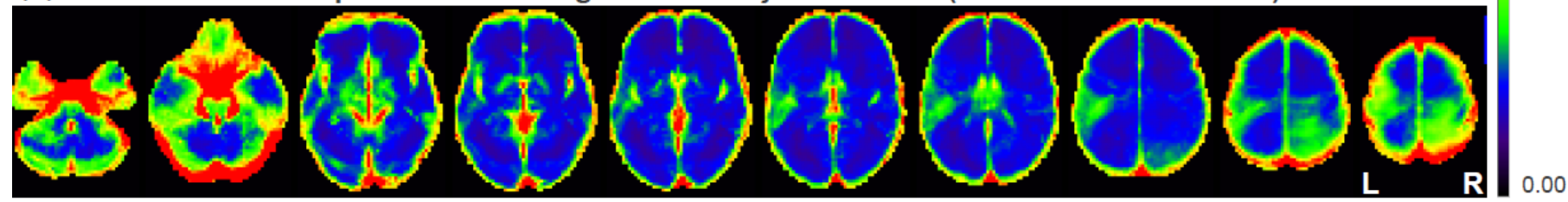

Figure 1: Parameter estimates of the homoscedastic and heteroscedastic model in the control group, computed from 35 healthy subjects. a) Mean perfusion estimate $\hat{\beta}_{\text {controls }}$. b) Combined within- and between-subject standard deviation estimate from the homoscedastic model $\hat{\sigma}_{G}$. c) Between-subject standard deviation estimate from the heteroscedastic model $\hat{\sigma}_{G}$. d) Root square of the average within-subject variance in the control group. Perfusion is expressed in normalized units (ratio to mean grey matter perfusion). Axial slices are displayed in neurological convention.

Values close to 1 mean that the within-subject variance $\left(\sigma_{s, v}^{2}\right)$ is preponderant compared to between-subject variance $\left(\sigma_{G, v}^{2}\right)$ and values close to 0 mean that betweensubject variance holds the majority of the total variance. This measure is provided as an output of the MEMA function in AFNI (Chen et al., 2012).

Constant within-subject variance across subjects. In a second experiment, we focused on the within-subject variance to verify whether it could be assumed roughly constant across subjects. To this aim, we calculated an average within-subject variance for each subject. This index was computed as suggested by Mumford and Nichols (2009) by averaging the within-subject variance for voxels within the interquartile range of the nonzero betweensubject variance. Given this index of within-subject variance, we searched for outliers in the control group with Rosner's test (Rosner, 1983), assuming that the distribution of the variance estimate was approximately normal. Then, we checked whether the control and patient groups had significantly different medians with a non-parametric Kruskal-Wallis test.

\subsubsection{Comparison of the homoscedastic and heteroscedastic models}

Detections at a fixed threshold. In order to assess the difference that heteroscedasticity modeling would induce, we compared the sensitivity and specificity of the homoscedastic and heteroscedastic models. We compared both approaches in a usual setting, where the detections were identified at a threshold $p<0.05$ with False Discovery Rate (FDR) correction for multiple comparisons Benjamini and Hochberg, 1995). The sensitivity was estimated as the average sensitivity across the patient group. In each group (patient, control), the specificity was estimated by averaging the specificity across subjects. As the data is usually pre-smoothed with a Gaussian kernel at the end of the pre-processing, we studied 6 kernel sizes within the typical smoothing range, defined by their full-width at half maximum (FWHM): $0 \mathrm{~mm}^{3}$ (i.e. no smoothing), $4 \mathrm{~mm}^{3}$, $6 \mathrm{~mm}^{3}, 8 \mathrm{~mm}^{3}, 10 \mathrm{~mm}^{3}, 12 \mathrm{~mm}^{3}$.

ROC analysis. We compared the sensitivity and specificity of the homoscedastic and heteroscedastic models with Receiver-Operating-Characteristics (ROC) curves. In order to draw the ROC curves, we used 122 p-values (un- 
corrected), equally spaced in the logarithmic space. For each p-value, the false positive rate (respectively true positive rate) was calculated as the average false positive rate (respectively true positive rate) over the subjects of the patient group. ROC curves provide a way to measure the performance of a classifier without focusing on a single threshold. We calculated the area under the curve as an indicator of the classification accuracy. Since the size of the expected detections is much smaller than the number of voxels that must not be detected, the area of interest in the ROC curve is the one of high specificity. That is why, as previously proposed in the literature, we focused on the area under the curve corresponding to false positive rates ranging from $0 \%$ to $10 \%$ (Skudlarski et al., 1999). As in the previous experiment, different smoothing kernels were studied.

\section{Results}

In this section we start with a graphical presentation of the parameter estimates of the homoscedastic and heteroscedastic models in the control group (5.1). Then, we present the results of the experiments regarding the validity of the assumptions of the homoscedastic model (5.2). In a last subsection, we display the results of the quantitative comparison between the homoscedastic and heteroscedastic models (5.3).

\subsection{ASL template: a model of normal perfusion}

In fig. 1, the parameter estimates of the homoscedastic and heteroscedastic models are displayed. Each parameter is defined as a voxelwise map. First, an estimate of the control group parameter, $\hat{\beta}_{\text {controls, }}$ is provided (the heteroscedastic estimate is displayed, the homoscedastic estimate being visually nearly identical). As expected, the $\mathrm{CBF}$ is higher in the cortex and the basal ganglia than in white matter. Then, three standard deviation estimates are displayed:

- the combined within- and between-subject standard deviation estimate $\hat{\sigma}_{G_{C}}$ from the homoscedastic model (1) b);

- the between-subject standard deviation estimate $\hat{\sigma}_{G}$ from the heteroscedastic model (1, c);

- the root square of the average within-subject variance estimates in the control group $\left(\frac{1}{n-1} \sum_{s=1}^{n-1} \hat{\sigma}_{s}^{2}\right)^{\frac{1}{2}}$. This map is not part of the estimated standard deviations but is provided as a visual example of expected within-subject standard deviation in the control group (1,d).

The high variance values observed in the vascular structures, such as the transverse sinus, are in concordance with the findings of Viviani et al. (2009). This pattern is clearly visible in the combined within- and betweensubject variance estimate of the homoscedastic model and

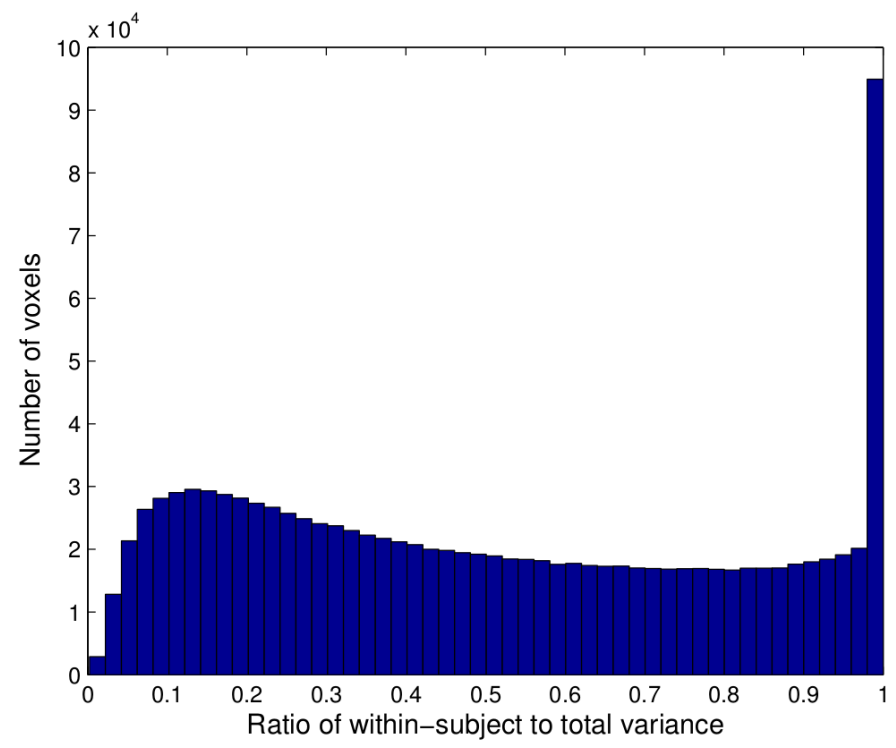

Figure 2: Histogram of the ratio of within-subject variance onto total variance in the control group across subjects and voxels. Both variance estimates have a significant impact depending on the voxels.

captured by the within-subject variance estimate in the heteroscedastic model. The main variations observed in the between-subject variance, as estimated in the heteroscedastic model, are related to inter-subject misregistrations in the cortex. An increased variance is also visible in the occipital lobe, probably related to increased arterial transit times in these regions (MacIntosh et al., 2010).

\subsection{Testing the assumptions of the homoscedastic model}

The homoscedastic model stands on one of the following assumptions: either the within-subject variance is negligible by comparison to between-subject variance, or the within-subject variance is roughly constant across subjects.

\subsubsection{Relative weights of between-subject and within-subject variances in the control group}

In order to verify if the within-subject variance can be assumed negligible by comparison to between-subject variance, fig. 2 presents the histogram of the ratio of withinsubject to total variance in the control group $\left(\lambda_{s, v}\right.$ from eq. (10)). Overall, a total of 1094790 voxels are considered. Values close to one indicate a preponderance of the within-subject variance, whereas values close to zero outline a dominating between-subject variance. Clearly, both components of variance have an important impact as the $\lambda_{s, v}$ index spans the complete range of values between 0 and 1 . The large peak indicating voxels with a zero between-subject variance was also observed in Chen et al. 2012) and might be the consequence of calculation inaccuracies. While the true between-subject variance might not be exactly zero, these voxels nevertheless present a very small between-subject variance, negligible by comparison 


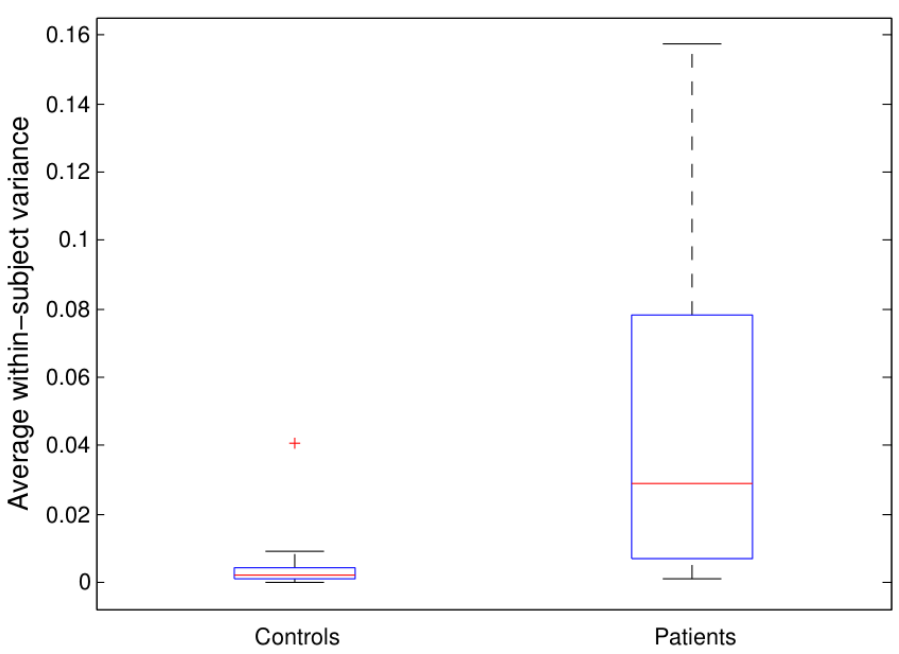

Figure 3: Average estimated within-subject variances in control and patient groups. For each box, the red line corresponds to the median and the top and bottom lines of the blue square are the upper and lower quartiles of the distribution. The whiskers cover the data points that are located up to 1.5 times the inter-quartile distance away from the lower or upper quartile. Points falling out of the whiskers are displayed with a red cross.

to the within-subject variance. The quartiles of the distribution are 0.23 and 0.78 , so that the outermost $25 \%$ of the voxels (on each side) have either a dominant within-subject variance or a dominant between-subject variance. In the remaining $50 \%$ voxels, both variance components have a significant impact. In conclusion, it cannot be assumed that within-subject variance is negligible in comparison to between-subject variance.

\subsubsection{Cross-subject comparison of within-subject variances}

In this experiment, we tested whether within-subject variance could be assumed roughly constant across subjects. As proposed by Mumford and Nichols (2009), we calculated an average within-subject variance across voxels. This led to an index of within-subject variance per subject. Fig. 3 displays the within-subject variance indexes in the control and patient groups.

In the control group, it is clear that one of the subjects presents an unexpected high variance by comparison to the other controls. Retrospectively, we found out that this subject moved substantially more than other controls and was identified as uncooperative by the MR physicist during the acquisition. As expected from fig. 3, we found one outlier in the control group with Rosner's test.

The within-subject variance was significantly higher in the patient group than in the control group $(p<0.05$ with Kruskal-Wallis test). This might be explained by the fact that patients tend to have more difficulties to lie still during the acquisition due to their pathological condition.

The index proposed by Mumford and Nichols (2009) is an average across voxels and therefore focused on global variations of the within-subject variance. However, strong variations, sometimes caused by artefacts, that appear lo- cally can also be a concern. As an example, fig. 4 a displays the estimated within-subject standard deviation for a control subject presenting locally atypical patterns. While the high variance induced by the presence of large vessels is a pattern shared across subjects (as previously described in fig. 1, last panel), the high variance observed bilaterally in the frontal lobe is specific of this control subject. We hypothesize that these strong variations are the consequence of motion during the acquisition that was not correctly compensated during the pre-processing. Even if this subject was not previously outlined as an outlier (with an average within-subject variance of 0.0024 ), these atypical patterns of variance might have a detrimental impact on the analysis if not properly taken into account.

In conclusion, variations across subjects of the withinsubject variance appear to be important in ASL. This might be a consequence of the low SNR of this technique, since small artefacts in the original control and labeled scans can lead to substantial variations in the perfusionweighted map after subtraction. In the next sections we investigate whether modeling heteroscedasticity can improve the detections of perfusion abnormalities.

\subsection{Comparison of homoscedastic and heteroscedastic models}

\subsubsection{Fixed threshold}

Quantitative analysis. Table 3 presents the sensitivity to detect hyper-perfusions and the specificity both in the patient group and by leave-one-out cross-validation in the control group with the homoscedastic and heteroscedastic models for different smoothing kernels (FWHM $=[0,4,6$, $\left.8,10,12] \mathrm{mm}^{3}\right)$, at $p<0.05$ FDR corrected.

Overall, the heteroscedastic model leads to a decrease in false positive rate. This is particularly noticeable in the patient group where the specificity is improved for each smoothing studied. As expected, in the control group, this effect is also observed but to a lesser extent. This is probably due to the fact that the hypothesis of homoscedasticity is better suited for the control subjects.

Qualitative analysis. At the individual level, as illustrated in fig. 4. local atypical patterns of variance can further lead to a substantial increase in false positives with the homoscedastic model. The unexpected high standard deviation in the frontal lobe for the control subject presented in fig. 4 leads to false positive hyper-perfusions with the homoscedastic model whereas the heteroscedastic model does not get any false positive (smoothing $\mathrm{FWHM}=8 \mathrm{~mm}^{3}$ ). Fig. 5 further illustrates the benefits of the heteroscedastic model compared to the homoscedastic model in a patient subject. The motion artefacts and hyper-signals induced by arteries indeed correspond to regions of high within-subject standard deviation. Modeling heterogeneous variances reduces the artefactual detections in these regions while preserving the quality of the true detections.

To investigate whether, at a same specificity rate, the sensitivity differs between the two approaches, we employed a ROC analysis as described in the next section. 
(a)

Within-subject standard deviation

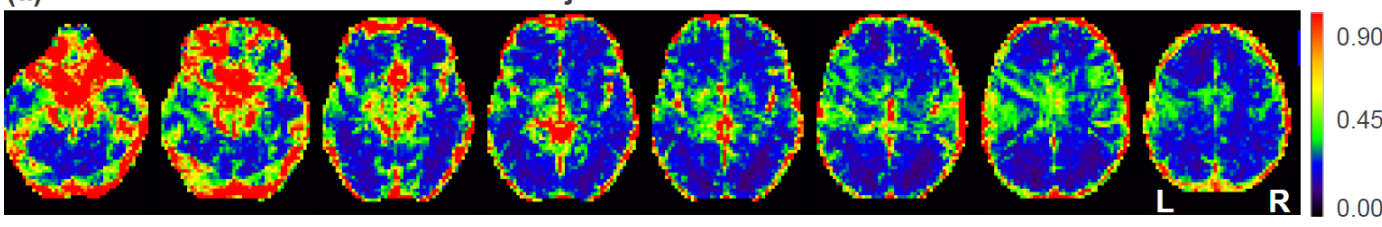

(b)

False positive detections (homoscedastic model)

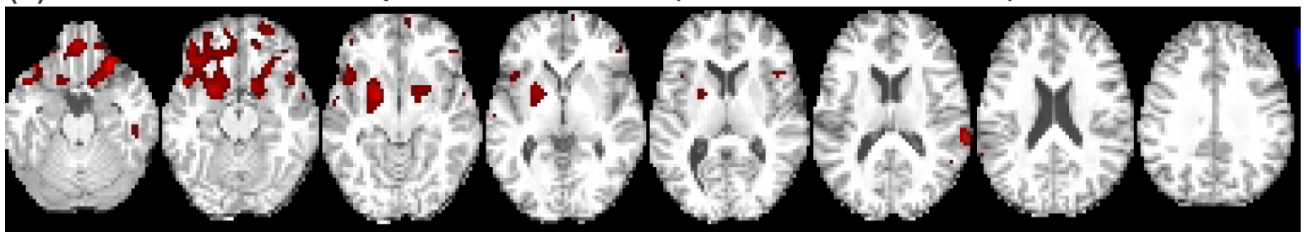

(c)

False positive detections (heteroscedastic model)

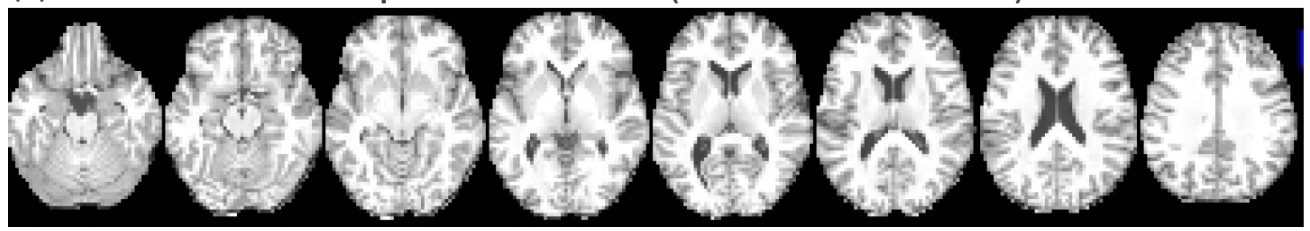

Figure 4: a) Estimated within-subject standard deviation in a control subject presenting locally atypical values. b) T1w map with false positive detections (in red) by leave-one-out cross-validation (smoothing FWHM $=8 \mathrm{~mm}^{3}$ ) with the homoscedastic model (b) and the heteroscedastic model (c). No false positive detections are observed with the heteroscedastic model.

Table 3: Sensitivity and specificity in the control (first row) and patient (second and third rows) groups with the homoscedastic and heteroscedastic models for different smoothing kernels (FWHM $=[0,4,6,8,10,12] \mathrm{mm}^{3}$ ), at $p<0.05$ FDR corrected.

\begin{tabular}{|c|c|c|c|c|c|c|c|c|c|c|c|c|}
\hline & \multicolumn{6}{|c|}{ Homoscedastic model } & \multicolumn{6}{|c|}{ Heteroscedastic model } \\
\hline & 0 & 4 & 6 & 8 & 10 & 12 & 0 & 4 & 6 & 8 & 10 & 12 \\
\hline Specificity (controls) & 1.00 & 0.99 & 0.99 & 0.99 & 0.99 & 0.99 & 1.00 & 1.00 & 1.00 & 1.00 & 1.00 & 1.00 \\
\hline Specificity (patients) & 0.92 & 0.89 & 0.87 & 0.86 & 0.85 & 0.84 & 1.00 & 0.99 & 0.99 & 0.99 & 0.99 & 0.98 \\
\hline Sensitivity (hyper) & 0.49 & 0.59 & 0.63 & 0.66 & 0.67 & 0.67 & 0.51 & 0.59 & 0.61 & 0.58 & 0.57 & 0.55 \\
\hline
\end{tabular}

\subsubsection{ROC analysis}

Quantitative comparison. Fig. 6 presents the ROC curves in the patient group for the homoscedastic and heteroscedastic models. The average over the studied smoothing kernels is plotted along with the spread provided by the standard deviation over the 6 measurements. The ROC curve of the heteroscedastic model is substantially closer to the ideal classifier than the homoscedastic curve.

Table 4 presents the area under the ROC curves for false positives rates ranging from 0 to $10 \%$, for the homoscedastic and heteroscedastic models with different smoothing kernels. The heteroscedastic model outperforms the homoscedastic model with an increased area under the curve. The best values are 0.72 and 0.49 with the heteroscedastic and homoscedastic model respectively. For both approaches, a maximum area under the curve is reached for a smoothing kernel of $8 \mathrm{~mm}^{3}$.

Qualitative comparison. In order to illustrate the advantage of the heteroscedastic over the homoscedastic model, we chose 3 representative subjects and compared the methods at fixed false positive rate and true positive rate. To this aim, we selected the uncorrected p-values that would lead to a pre-specified false positive rate (respectively true positive rate) from the ROC analysis. We worked with data smoothed with a Gaussian kernel of $8 \mathrm{~mm}^{3}$ that led to the best area under the curve with both models. It is worth noting that in the previous section the sensitivity and specificity were estimated at the group level and that the 3 subjects presented in this part were chosen so that they would best illustrate the group findings. Fig. 7 presents the detections obtained with both methods on the 3 selected patients. The first subject, which presents a small hyper-perfusion, is studied at a true positive rate of $50 \%$. In the two remaining patients, the methods are compared at a false positive rate of $0.1 \%$.

Patient 15 suffers from a gliosarcoma in the left hemisphere close to the parahippocampal region. The lesion displays a small hyper-perfusion in its dorsal part, small hypo-perfusions are seen in the surrounding edema. At a true positive rate of $50 \%$, the homoscedastic model displays a larger number of false positives than the heteroscedastic model. Patient 16 was diagnosed with a high grade tumor in the left temporal lobe. The lesion is characterized by a large hyper-perfusion and a surrounding hypo-perfusion. With a false positive rate of $0.1 \%$, the hyper-perfusion is correctly located with both methods. The extent of the hyper-perfusion is however better cov- 
Table 4: Area under the ROC curve for false positive rates ranging between $0 \%$ and $10 \%$ with the homoscedastic and heteroscedastic models. The heteroscedastic model outperforms the homoscedastic model.

\begin{tabular}{|c|c|c|c|c|c|c|c|c|c|c|c|c|}
\hline & \multicolumn{6}{|c|}{ Homoscedastic model } & \multicolumn{6}{|c|}{ Heteroscedastic model } \\
\hline & 0 & 4 & 6 & 8 & 10 & 12 & 0 & 4 & 6 & 8 & 10 & 12 \\
\hline Area under the ROC curve & 0.46 & 0.49 & 0.49 & 0.49 & 0.48 & 0.48 & 0.63 & 0.70 & 0.72 & 0.72 & 0.69 & 0.65 \\
\hline
\end{tabular}
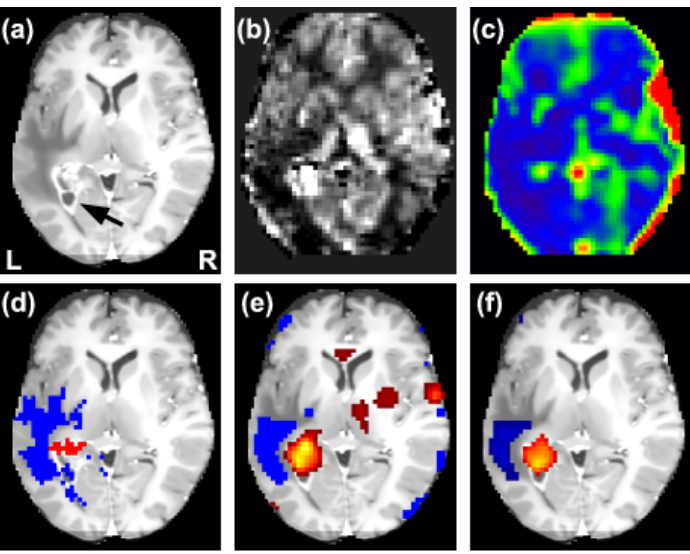

Figure 5: Detections of perfusion abnormalities with the homoscedastic and heteroscedastic models in a patient suffering from a high grade glioma. a) T1w-Gd map, the tumor site is pointed by a black arrow. b) Patient ASL CBF estimate $\hat{\beta}_{n}$. c) Within-subject standard deviation of ASL CBF $\hat{\sigma}_{n}^{2}$. d) T1w-Gd map with ground truth overlaid. T1w-Gd map with hypo- (blue color-map) and hyper-perfusions (hot color-map) overlaid for the homoscedastic (e) and heteroscedastic (f) models. Modeling heterogeneous variances (heteroscedastic model) reduces the false positive detections while preserving the true detections. Axial slices are displayed in neurological convention.

ROC curves of the homoscedastic and heteroscedastic models

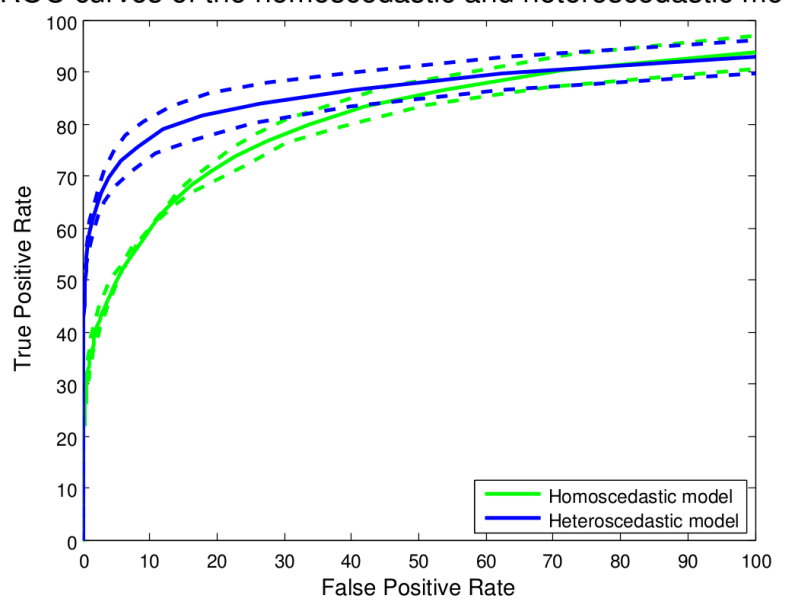

Figure 6: ROC curves for perfusion abnormality detections with the homoscedastic and heteroscedastic models. The average ROC curves across the studied smoothings are plotted in plain line. Dotted lines are plotted one standard deviation away from the average.

ered by the heteroscedastic model. Patient 6 suffers from a meningioma partly hyperperfused. Similarly to patient 16 , at a false positive rate of $0.1 \%$, both methods detect the hyper-perfusion but the heteroscedastic model is clearly more sensitive.

These 3 cases illustrate the loss of sensitivity of the homoscedastic model by comparison to the heteroscedastic model at the same specificity level.

\section{Discussion and conclusion}

We have compared two approaches to quantitatively outline patient-specific pathological patterns of abnormal perfusion in ASL data based on the massively univariate GLM: the homoscedastic and heteroscedastic models.

We demonstrated that the assumptions underlying the homoscedastic model are not verified in ASL studies. More precisely, the within-subject variance cannot be considered as negligible by comparison to between-subject variance, nor constant across subjects. In fMRI data, small deviations from homoscedasticity have shown to not overly alter the results in one-sample mixed-effects GLM analysis (Mumford and Nichols, 2009). Here, we showed that modeling heterogeneous within-subject variances is essential in order to reach a satisfactory level of specificity in a mixed-effect two-sample t-test comparing a patient to a group of controls in ASL. These results are in line with a recent study by Chen et al. (2012) where heteroscedastic mixed-effects GLM were shown to provide more accurate results in fMRI.

In the context of this paper, we defined a single parameter in the subject-level design matrix and focused on perfusion-weighted images obtained after pair-wise subtraction of the control and labeled scans. Other authors have suggested that taking the complete ASL time-course into account (before subtraction) would lead to more efficient estimates of perfusion in fMRI (Mumford et al. 2006). Also, additional regressors can be introduced as nuisance covariates for denoising purposes in the subjectlevel design matrix as suggested in (Wang, 2012). Modifying the subject-level design matrix would change the estimated subject parameters and their sampling variances. However, an heteroscedastic model would still be appropriate in this setting so that the conclusions of this paper remain valid even with a different subject-level model.

We demonstrated that within-subject variance captures important information regarding the subject-specific spatial distribution of noise in ASL data. We also outlined that patient-specific brain perfusion abnormalities can be correctly detected using ASL if the heterogeneous withinsubject variances are properly modeled. We therefore advise the use of heteroscedastic models in ASL studies. 


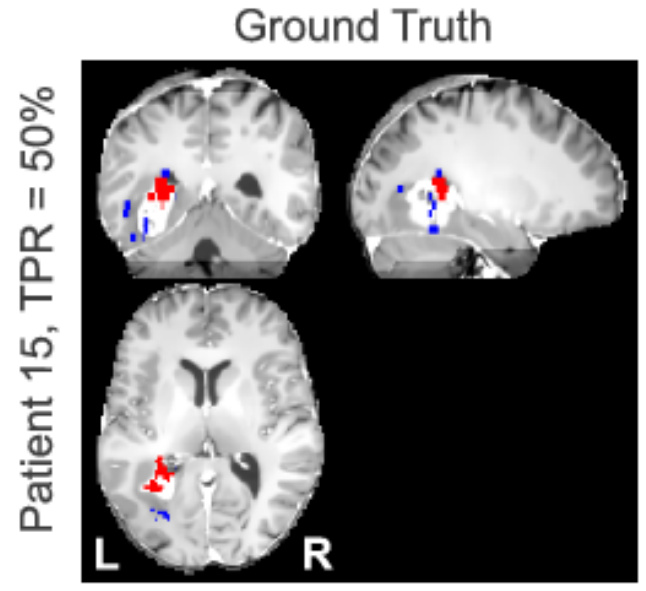

Homoscedastic model
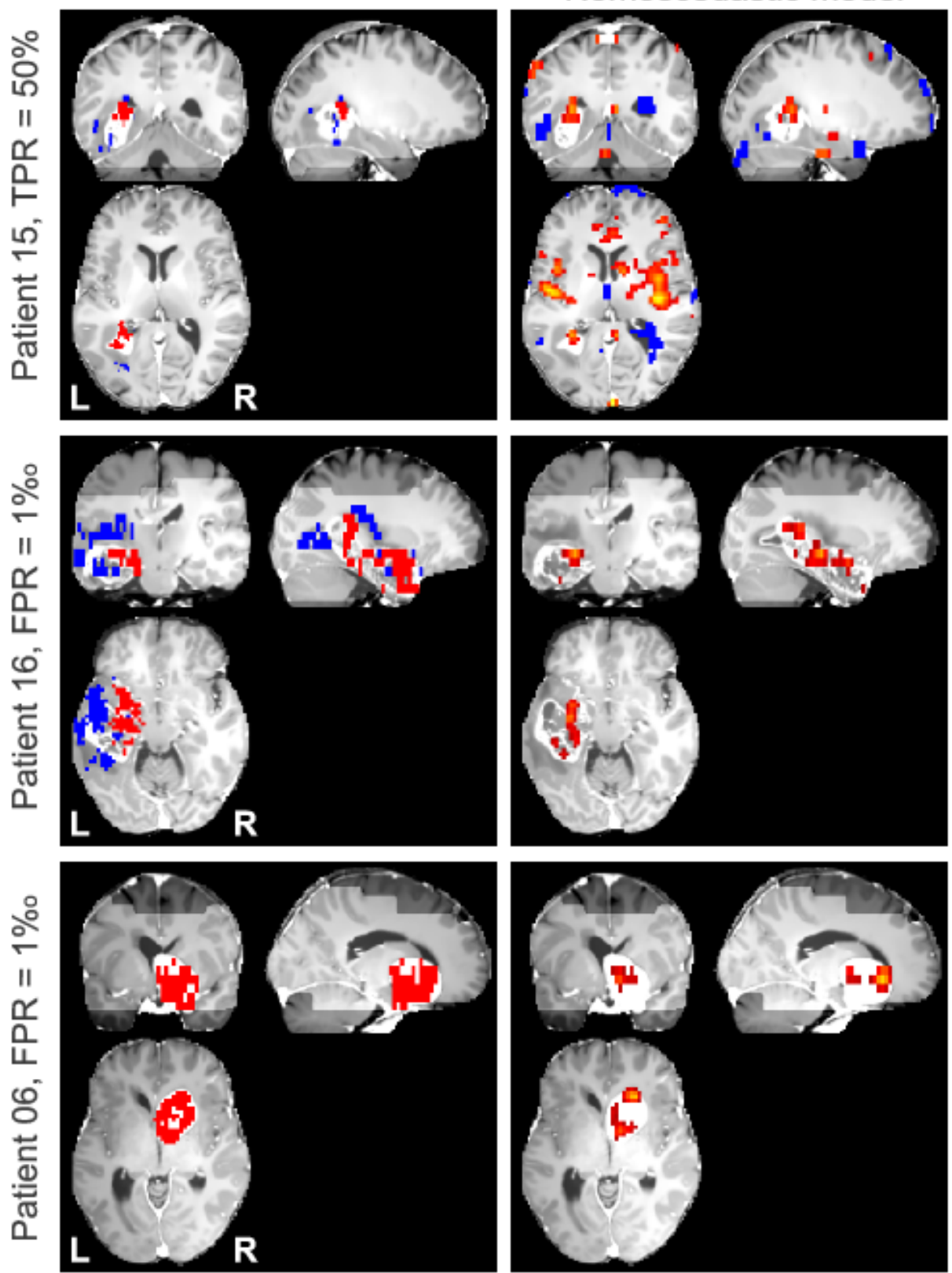
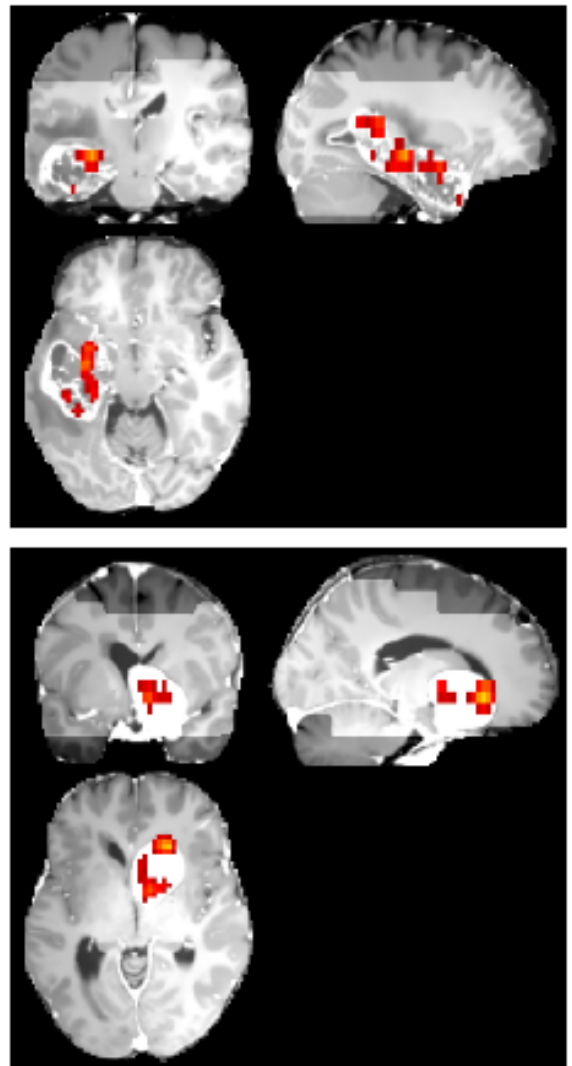

Heteroscedastic model
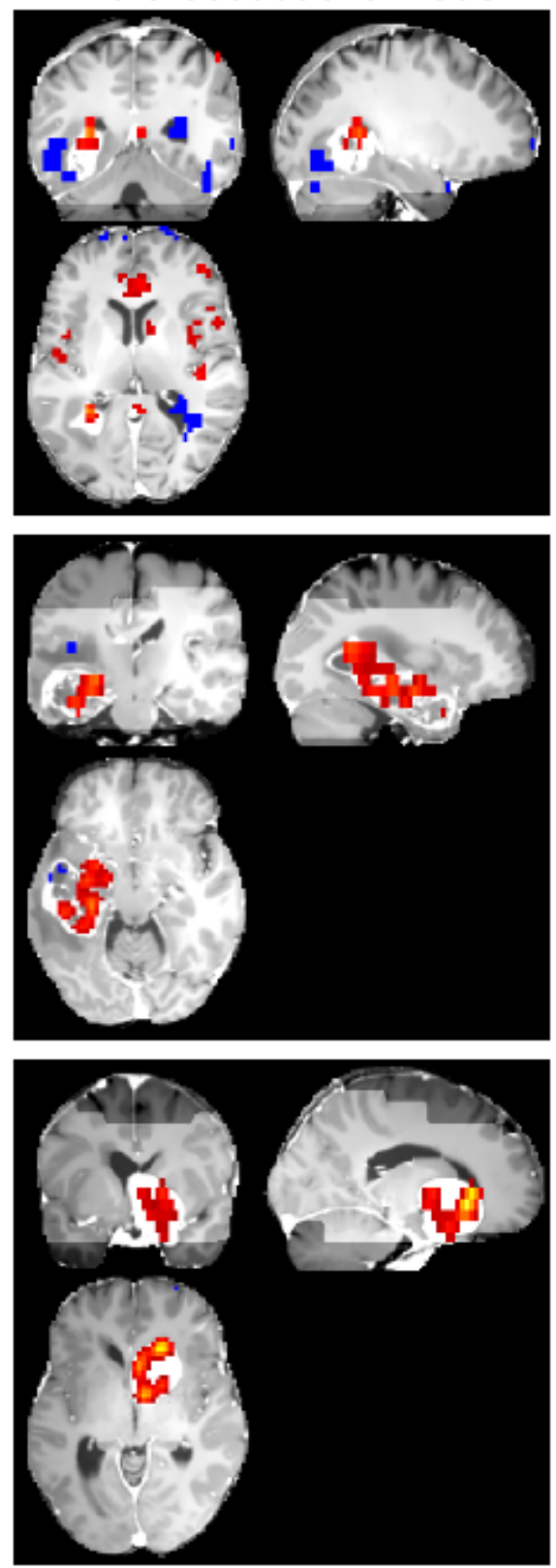

Figure 7: Perfusion abnormalities detections in 3 patients with homoscedastic and heteroscedastic models. From left to right: ground truth hyper-perfusions (red) and hypo-perfusions (blue) overlaid on the T1w-Gd map; detections with the homoscedastic model with a smoothing kernel of FWHM $=8 \mathrm{~mm}^{3}$, hyper-perfusions (hot colormap) and hypo-perfusions (blue); detections with the heteroscedastic model with a smoothing kernel of FWHM $=8 \mathrm{~mm}^{3}$, hyper-perfusions (hot colormap) and hypo-perfusions (blue). 


\section{References}

Aguirre, G.K., Detre, J.a., Zarahn, E., Alsop, D.C., 2002. Experimental design and the relative sensitivity of BOLD and perfusion fMRI. NeuroImage 15, 488-500.

Aguirre, G.K., Zarahn, E., D'Esposito, M., 1997. Empirical analyses of BOLD fMRI statistics. II. Spatially smoothed data collected under null-hypothesis and experimental conditions. NeuroImage 5, 199-212.

Ashburner, J., Friston, K.J., 2005. Unified segmentation. NeuroImage $26,839-851$

Aslan, S., Lu, H., 2010. On the sensitivity of ASL MRI in detecting regional differences in cerebral blood flow. Magnetic Resonance Imaging 28, 928-935.

Beckmann, C.F., Jenkinson, M., Smith, S.M., 2003. General multilevel linear modeling for group analysis in FMRI. NeuroImage 20, 1052-1063.

Benjamini, Y., Hochberg, Y., 1995. Controlling the False Discovery Rate: A Practical and Powerful Approach to Multiple Testing. Journal of the Royal Statistical Society. Series B (Methodological) 57, 289-300.

Buxton, R.B., Frank, L.R., Wong, E.C., Siewert, B., Warach, S., Edelman, R.R., 1998. A general kinetic model for quantitative perfusion imaging with arterial spin labeling. Magnetic Resonance in Medicine 40, 383-396.

Chen, G., Saad, Z.S., Nath, A.R., Beauchamp, M.S., Cox, R.W., 2012. FMRI group analysis combining effect estimates and their variances. NeuroImage 60, 747-765.

Cox, R.W., 1996. AFNI: software for analysis and visualization of functional magnetic resonance neuroimages. Computers and biomedical research, an international journal 29, 162-73.

Ferré, J.C., Petr, J., Bannier, E., Barillot, C., Gauvrit, J.Y., 2012. Improving quality of arterial spin labeling MR imaging at 3 tesla with a 32-channel coil and parallel imaging. Journal of Magnetic Resonance Imaging 1239, 1233-1239.

Friston, K.J., Stephan, K.E., Lund, T.E., Morcom, A., Kiebel, S. 2005. Mixed-effects and fMRI studies. NeuroImage 24, 244-52.

Holmes, A., Friston, K., 1998. Generalisability, Random Effects \& Population Inference. Proceedings of Fourth International Conference on Functional Mapping of the Human Brain, NeuroImage 7, S754 .

Lindquist, M.a., Spicer, J., Asllani, I., Wager, T.D., 2012. Estimating and testing variance components in a multi-level GLM. NeuroImage 59, 490-501.

MacIntosh, B.J., Filippini, N., Chappell, M.a., Woolrich, M.W., Mackay, C.E., Jezzard, P., 2010. Assessment of arterial arrival times derived from multiple inversion time pulsed arterial spin labeling MRI. Magnetic Resonance in Medicine 63, 641-647.

Mériaux, S., Roche, A., Dehaene-Lambertz, G., Thirion, B., Poline, J.B., 2006. Combined permutation test and mixed-effect model for group average analysis in fMRI. Human brain mapping 27, 402-10.

Mumford, J.a., Hernandez-Garcia, L., Lee, G.R., Nichols, T.E., 2006. Estimation efficiency and statistical power in arterial spin labeling fMRI. NeuroImage 33, 103-14.

Mumford, J.A., Nichols, T., 2009. Simple group fMRI modeling and inference. NeuroImage 47, 1469-1475.

Noguchi, T., Yoshiura, T., Hiwatashi, A., Togao, O., Yamashita, K., Nagao, E., Shono, T., Mizoguchi, M., Nagata, S., Sasaki, T., Suzuki, S.O., Iwaki, T., Kobayashi, K., Mihara, F., Honda, H., 2008. Perfusion imaging of brain tumors using arterial spinlabeling: correlation with histopathologic vascular density. American Journal of Neuroradiology 29, 688-93.

Ostergaard, L., Weisskoff, R.M., Chesler, D.A., Gyldensted, C. Rosen, B.R., 1996. High resolution measurement of cerebral blood flow using intravascular tracer bolus passages. Part I: Mathematical approach and statistical analysis. Magnetic Resonance in Medicine 36, 715-725.

Penny, W.D., Holmes, A.J., 2004. Random-Effects Analysis, in: Elsevier (Ed.), Human Brain Function. San Diego, pp. 843-850.

Petr, J., Ferré, J.C., Raoult, H., Bannier, E., Gauvrit, J.Y., Barillot, C., 2012. Template-based approach for detecting motor task activation-related hyperperfusion in pulsed asl data. Human Brain Mapping

Poldrack, R.A., Mumford, J., Nichols, T., 2011. Handbook of functional MRI data analysis. Cambridge University Press, Cambridge.

Rosner, B., 1983. Percentage points for a generalized ESD manyoutlier procedure. Technometrics 25, 165-172.

Skudlarski, P., Constable, R.T., Gore, J.C., 1999. ROC analysis of statistical methods used in functional MRI: individual subjects. NeuroImage 9, 311-329.

Thirion, B., Pinel, P., Mériaux, S., Roche, A., Dehaene, S., Poline, J.B., 2007. Analysis of a large fMRI cohort: Statistical and methodological issues for group analyses. NeuroImage 35, 105-20. Viviani, R., Sim, E.J., Lo, H., Richter, S., Haffer, S., Osterfeld, N., Thöne, J., Beschoner, P., 2009. Components of variance in brain perfusion and the design of studies of individual differences: the baseline study. NeuroImage 46, 12-22.

Wang, Y., Saykin, A.J., Pfeuffer, J., Lin, C., Mosier, K.M., Shen, L., Kim, S., Hutchins, G.D., 2011. Regional reproducibility of pulsed arterial spin labeling perfusion imaging at 3T. NeuroImage 54, 1188-1195.

Wang, Z., 2012. Improving cerebral blood flow quantification for arterial spin labeled perfusion MRI by removing residual motion artifacts and global signal fluctuations. Magnetic resonance imaging .

Wintermark, M., Sesay, M., Barbier, E., Borbély, K., Dillon, W.P., Eastwood, J.D., Glenn, T.C., Grandin, C.B., Pedraza, S., Soustiel, J.F., Nariai, T., Zaharchuk, G., Caillé, J.M., Dousset, V., Yonas, H., 2005. Comparative overview of brain perfusion imaging techniques. Stroke 36, e83-e99.

Wong, E.C., Buxton, R.B., Frank, L.R., 1998. Quantitative imaging of perfusion using a single subtraction (QUIPSS and QUIPSS II). Magnetic Resonance in Medicine 39, 702-708.

Woolrich, M.W., Behrens, T.E.J., Beckmann, C.F., Jenkinson, M., Smith, S.M., 2004. Multilevel linear modelling for FMRI group analysis using Bayesian inference. NeuroImage 21, 1732-47.

Worsley, K.J., Liao, C.H., Aston, J., Petre, V., Duncan, G.H., Morales, F., Evans, a.C., 2002. A general statistical analysis for fMRI data. NeuroImage 15, 1-15.

Zaharchuk, G., Bammer, R., Straka, M., Shankaranarayan, A., Alsop, D.C., Fischbein, N.J., Atlas, S.W., Moseley, M.E., 2009. Arterial spin-label imaging in patients with normal bolus perfusionweighted MR imaging findings: pilot identification of the borderzone sign. Radiology 252, 797-807. 